\title{
Generalised energy conservation law for chaotic phenomena
}

\author{
Jing-Tang Xing ${ }^{1}$ \\ Received: 19 March 2019 / Revised: 9 May 2019 / Accepted: 4 June 2019 / Published online: 22 July 2019 \\ (c) The Author(s) 2019
}

\begin{abstract}
Chaotic phenomena are increasingly being observed in all fields of nature, where investigations reveal that a natural phenomenon exhibits nonlinearities and attempts to reveal their deep underlying mechanisms. Chaos is normally understood as "a state of disorder", for which there is as yet no universally accepted mathematical definition. A commonly used concept states that, for a dynamical system to be classified as chaotic, it must have the following properties: be sensitive to initial conditions, show topological transitivity, have densely periodical orbits etc. Revealing the rules that govern chaotic motion is thus an important unsolved task for exploring nature. We present herein a generalised energy conservation law governing chaotic phenomena. Based on two scalar variables, viz. generalised potential and kinetic energies defined in the phase space describing nonlinear dynamical systems, we find that chaotic motion is periodic motion with infinite time period whose time-averaged generalised potential and kinetic energies are conserved over its time period. This implies that, as the averaging time is increased, the time-averaged generalised potential and kinetic energies tend to constants while the time-averaged energy flows, i.e., their rates of change with time, tend to zero. Numerical simulations on reported chaotic motions, such as the forced van der Pol system, forced Duffing system, forced smooth and discontinuous oscillator, Lorenz's system, and Rössler's system, show the above conclusions to be correct according to the results presented herein. This discovery may indicate that chaotic phenomena in nature could be controlled because, even though their instantaneous states are disordered, their long-time averages can be predicted.
\end{abstract}

Keywords Chaos · Generalised energy $\cdot$ Generalised energy conservation $\cdot$ Nonlinear dynamical systems $\cdot$ Energy flow theory

\section{Nonlinear dynamical systems}

For this work on nonlinear dynamic chaotic systems, it is generally sufficient to consider a second-order differential equation with its initial conditions, which can be transformed into a first-order differential equation in phase space with the following non-dimensional form [1]:

$\mathrm{d} \boldsymbol{y} / \mathrm{d} t \equiv \dot{\boldsymbol{y}}=\boldsymbol{f}(t, \boldsymbol{y}), \quad \boldsymbol{y}(0)=\boldsymbol{y}_{0}$.

Generally, we consider that $\boldsymbol{y}=\boldsymbol{y}(t) \in R^{n}$ is a vectorvalued function of an independent variable $t \in I=\left(t_{1}, t_{2}\right) \subseteq$ $R$ and $f: U \rightarrow R^{n}$ is a smooth function of the variable $t$ and the vector $\boldsymbol{y}$ defined on some subset $U \subseteq R^{n}$, an $n$ -

Jing-Tang Xing

jtxing@soton.ac.uk

1 Faculty of Engineering and Physical Sciences, University of Southampton, Southampton SO17 1BJ, UK dimensional phase space. Often, one seeks a solution $\varphi\left(\boldsymbol{y}_{0}, t\right)$ such that

$\varphi\left(y_{0}, 0\right)=y_{0}$

The solution $\varphi\left(y_{0}, \cdot\right): I \rightarrow R^{n}$ defines a solution curve, i.e., trajectory or orbit, of Eq. (1) based at $\boldsymbol{y}_{0}$, as shown in Fig. 1a. According to the local existence and uniqueness theorems of solutions [2], no intersections of the trajectories of Eq. (1) exist in the solution space, except at its fixed points. All possible solution curves $\varphi_{t}(U)$ generate the flow shown in Fig. 1b.

\section{Energy flow variables and equations}

To investigate the behaviours of nonlinear dynamical systems, based on Eq. (1) in phase space, Xing [3] introduced three scalar energy flow variables:

generalised potential energy (GPE): $E=\boldsymbol{y}^{\mathrm{T}} \boldsymbol{y} / 2$, 


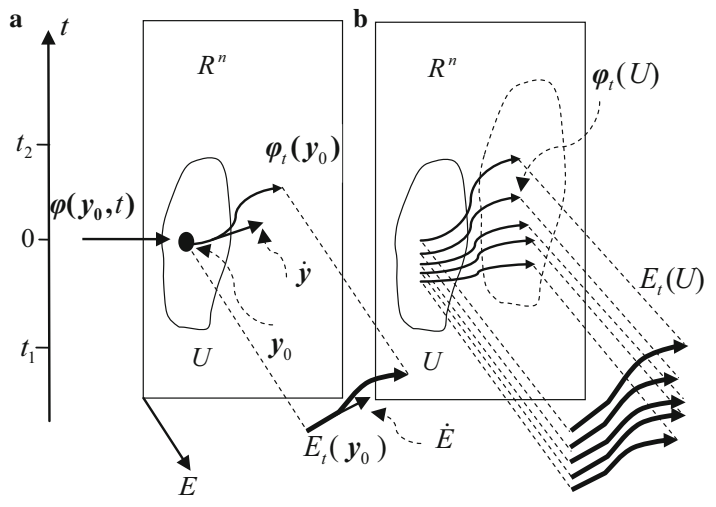

Fig. 1 a Solution curve $\varphi_{t}\left(y_{0}\right)$ with its energy flow curve $E_{t}\left(y_{0}\right)$, of which the tangent vectors at a point $\boldsymbol{y}$ are $\dot{\boldsymbol{y}}=\boldsymbol{f}$ and $\dot{E}$, respectively. b Flow $\varphi_{t}(U)$ and energy flow $E_{t}(U)$ in $R^{n}$

generalised kinetic energy (GKE): $K=\dot{\boldsymbol{y}}^{\mathrm{T}} \dot{\boldsymbol{y}} / 2$,

generalised force power: $P=\boldsymbol{y}^{\mathrm{T}} \boldsymbol{f}$,

whose time-averaged values during a time period $(0, T)$ are defined respectively by

$$
\begin{aligned}
& \langle E\rangle=\int_{0}^{T} E \mathrm{~d} t / T, \\
& \langle K\rangle=\int_{0}^{T} K \mathrm{~d} t / T, \\
& \langle P\rangle=\int_{0}^{T} P \mathrm{~d} t / T .
\end{aligned}
$$

The corresponding energy flow equilibrium equations take the forms

$\dot{E}=P, \quad E_{0}=\boldsymbol{y}_{0}^{\mathrm{T}} \boldsymbol{y}_{0} / 2$,

$\dot{K}=\dot{\boldsymbol{y}}^{\mathrm{T}}(\partial \boldsymbol{f} / \partial t+\boldsymbol{J} \dot{\boldsymbol{y}})=\dot{\boldsymbol{y}}^{\mathrm{T}} \partial \boldsymbol{f} / \partial t+\dot{\boldsymbol{y}}^{\mathrm{T}} \boldsymbol{E} \dot{\boldsymbol{y}}$,

$K_{0}=\dot{\boldsymbol{y}}_{0}^{\mathrm{T}} \dot{\boldsymbol{y}}_{0} / 2, \quad \boldsymbol{J}=\partial \boldsymbol{f} / \partial \boldsymbol{y}^{\mathrm{T}}$,

$\boldsymbol{E}=\left(\boldsymbol{J}+\boldsymbol{J}^{\mathrm{T}}\right) / 2, \quad \boldsymbol{U}=\left(\boldsymbol{J}-\boldsymbol{J}^{\mathrm{T}}\right) / 2$,

where $\boldsymbol{J}$ denotes the Jacobian matrix, $\boldsymbol{E}$ is a real symmetrical matrix called the energy flow matrix, and $\boldsymbol{U}$ is a skew-symmetrical matrix, such that $\dot{\boldsymbol{y}}^{\mathrm{T}} \boldsymbol{U} \dot{\boldsymbol{y}} \equiv 0$. The timeaveraged energy flows are defined as

$$
\begin{aligned}
& \langle\dot{E}\rangle=\int_{0}^{T} \dot{E} \mathrm{~d} t / T=[E(T)-E(0)] / T, \\
& \left\langle E_{0}\right\rangle=\left\langle\boldsymbol{y}_{0}^{\mathrm{T}} \boldsymbol{y}_{0}\right\rangle / 2, \\
& \langle\dot{K}\rangle=\int_{0}^{T} \dot{K} \mathrm{~d} t / T=[K(T)-K(0)] / T, \\
& \left\langle K_{0}\right\rangle=\left\langle\dot{\boldsymbol{y}}_{0}^{\mathrm{T}} \dot{\boldsymbol{y}}_{0}\right\rangle / 2 .
\end{aligned}
$$

Geometrically, $E$ is related to the position $y$ of a point in phase space, while $K$ involves the velocity or tangent vector $\dot{\boldsymbol{y}}$ of the solution curve, and the generalised force power $P$ gives the energy flow, i.e., the rate of change of $E$ with time. As shown in Fig. 1, an axis for $E$ is given, from which the potential energy flow (PEF) curves are drawn.

\section{Energy flow characteristic factors and vectors}

The real symmetric energy flow matrix $\boldsymbol{E}$ has real eigenvalues $\lambda_{I}$ and eigenvectors $\psi_{I}$ satisfying the orthogonal relationships

$$
\begin{aligned}
& \boldsymbol{\Psi}^{\mathrm{T}} \boldsymbol{E} \boldsymbol{\Psi}=\boldsymbol{\Lambda}=\operatorname{diag}\left(\lambda_{I}\right), \quad \boldsymbol{\Psi}^{\mathrm{T}} \boldsymbol{\Psi}=\boldsymbol{I}, \\
& \boldsymbol{\Psi}=\left[\boldsymbol{\psi}_{1} \boldsymbol{\psi}_{2} \cdots \boldsymbol{\psi}_{n}\right] .
\end{aligned}
$$

These eigenvectors span a complete subspace in the neighbourhood of the point where the matrix $\boldsymbol{E}$ is defined, so that the variation vector $\boldsymbol{\varepsilon}$ and a real quantity $\boldsymbol{\varepsilon}^{\mathrm{T}} \boldsymbol{E} \boldsymbol{\varepsilon}$ around this point can be represented as

$\boldsymbol{\varepsilon}=\boldsymbol{\Psi} \zeta \boldsymbol{\varepsilon}^{\mathrm{T}} \boldsymbol{E} \boldsymbol{\varepsilon}=\boldsymbol{\zeta}^{\mathrm{T}} \boldsymbol{\Lambda} \zeta=\sum_{I=1}^{n} \lambda_{I} \zeta_{I}^{2}$

This implies that $\boldsymbol{\varepsilon}^{\mathrm{T}} \boldsymbol{E} \boldsymbol{\varepsilon}$ is totally determined by the eigenvalues of the energy flow matrix, thus we call $\lambda_{I}$ and $\psi_{I}$ the energy flow characteristic factors and the energy flow characteristic vectors of the nonlinear dynamical system, respectively.

\section{Phase volume strain and its time rate of change}

As shown in Fig. 2, consider a phase space volume $V$ closed by a surface $S$ with unit outside normal $v_{i}$, which moves to its new position represented by a dashed line due to a displacement $\dot{y} \Delta t$ caused by the flow of points on the surface $S$ in a time interval $\Delta t$. Integrating the divergence of the vector $f$ over the volume $V$ and using the summation convention and Green's theorem [4], we obtain

$\int_{V} f_{i, i} \mathrm{~d} V=\int_{V} \dot{y}_{i, i} \mathrm{~d} V=\int_{S} \dot{y}_{i} v_{i} \mathrm{~d} S=\frac{\Delta V}{\Delta t}$,

where $\Delta V$ represents the change of volume $V$ in the time interval $\Delta t$. Equation (10) is valid for any size of volume $V$, so we can consider a differential volume element $V_{0}$ and Eq. (10) is approximated to

$V_{0} f_{i, i}=\mathrm{D} V / \mathrm{D} t, f_{i, i}=\mathrm{D} V /\left(V_{0} \mathrm{D} t\right)=\dot{v}$,

$v=\left(V-V_{0}\right) / V_{0}$, 


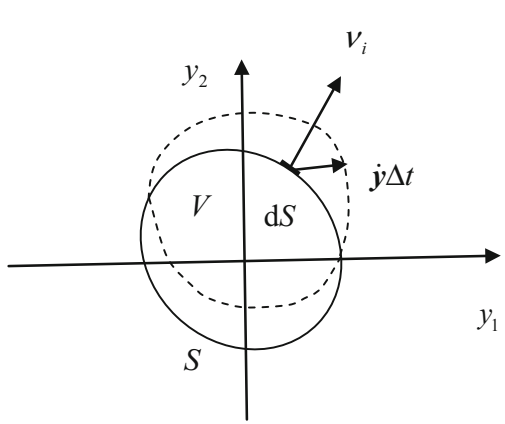

Fig. 2 Integration of the divergence of a vector field over a phase space volume

where $v$ and $\dot{v}$ are defined as the phase volume stain and its time rate of change. Generally, these are functions of the phase point and time. From Eqs. (6) and (11), it follows that

$f_{i, i}=\operatorname{tr}(\boldsymbol{J})=\operatorname{tr}(\boldsymbol{E})=\sum_{I=1}^{n} \lambda_{I}, \quad \dot{v}=\sum_{I=1}^{n} \lambda_{I}$.

Equation (12) indicates that the time rate of change of the phase volume strain equals the summation of the energy flow characteristic factors of the nonlinear dynamical system, from which we have

$\begin{cases}\dot{v}<0, & \sum_{I=1}^{n} \lambda_{I}<0, \\ \dot{v}=0, & \sum_{I=1}^{n} \lambda_{I}=0, \\ \dot{v}>0, & \sum_{I=1}^{n} \lambda_{I}>0\end{cases}$

which respectively correspond to a contracting, isovolumetric, and expanding phase space for the nonlinear dynamical system around the point where $\boldsymbol{E}$ is defined.

\section{Zero-energy flow surface, fixed points and stabilities}

Generally, the energy flow of a system is a function of time and the position of a point in phase space, which generates a scalar field called the energy flow field of the nonlinear dynamical system. The equation

$\dot{E}=\boldsymbol{y}^{\mathrm{T}} \boldsymbol{f}(t, \boldsymbol{y})=P(t, \boldsymbol{y})=0$

defines a generalised surface or subspace in the phase space, called a zero-energy flow surface, on which the energy flow vanishes. If an orbit of the nonlinear dynamical system is on a zero-energy flow surface, the distance of a point on the orbit does not change with time. There are three cases satisfying $\dot{E}=0$ in Eq. (14):

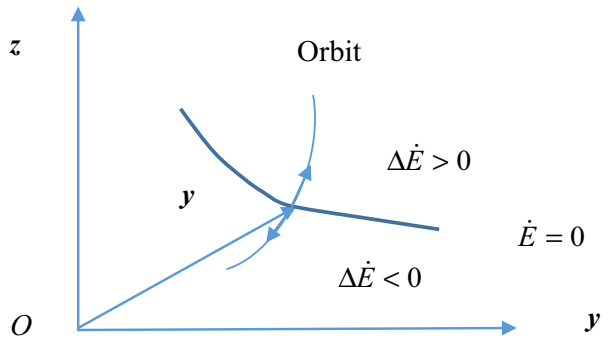

Fig. 3 Zero-energy flow surface and an unstable point $y$ at the intersection of the orbit and this surface, determined by $\Delta \dot{E}$

- Case $1 \boldsymbol{y}=\mathbf{0}$, representing the origin of phase space, at which $E$ is defined as zero.

- Case $2 f=\mathbf{0}$, implying an equilibrium point of the system.

- Case $3 P=0, \quad \boldsymbol{y} \neq \mathbf{0} \neq \boldsymbol{f}$, corresponding to a $\dot{E}=0$ surface.

Assuming that $\boldsymbol{y}$ denotes an orbit point on a zero-energy flow surface, $P(t, \boldsymbol{y})=0$, and that $\boldsymbol{\varepsilon}$ is a small orbit variation around $\boldsymbol{y}$, generally the variation of the energy flow caused by the orbit variation is given by

$\Delta \dot{E}=\boldsymbol{\varepsilon}^{\mathrm{T}} \boldsymbol{p}+\boldsymbol{\varepsilon}^{\mathrm{T}} \boldsymbol{E} \boldsymbol{\varepsilon}, \quad \boldsymbol{p}=\boldsymbol{f}+\boldsymbol{J}^{\mathrm{T}} \boldsymbol{y}$,

which reduces to

$\Delta \dot{E}=\boldsymbol{\varepsilon}^{\mathrm{T}} \boldsymbol{E} \boldsymbol{\varepsilon}$

for the equilibrium point $\boldsymbol{y}=\mathbf{0}$ or the case $\boldsymbol{\varepsilon}^{\mathrm{T}} \boldsymbol{p}=0$. The vector $\boldsymbol{p}$ is an energy flow gradient along the normal vector of the zero-energy flow surface, thus $\boldsymbol{\varepsilon}^{\mathrm{T}} \boldsymbol{p}=0$ implies that the vector $\varepsilon$ is on the surface and perpendicular to the vector p.

Based on Eq. (15) and the geometrical meaning of potential energy, we conclude that:

- if $|\boldsymbol{y}+\boldsymbol{\varepsilon}|<|\boldsymbol{y}|$, then $E(t, \boldsymbol{y}+\boldsymbol{\varepsilon})<E(t, \boldsymbol{y})$, so $\Delta \dot{E}>0$ implies a flow towards the zero-energy flow surface, while $\Delta \dot{E}<0$ indicates a flow backwards from the zero-energy flow surface;

- if $|\boldsymbol{y}+\boldsymbol{\varepsilon}|>|\boldsymbol{y}|$, then $E(t, \boldsymbol{y}+\boldsymbol{\varepsilon})>E(t, \boldsymbol{y})$, so $\Delta \dot{E}<0$ implies a flow towards the zero-energy flow surface, while $\Delta \dot{E}>0$ indicates a flow backwards from the zero-energy flow surface;

- if the flows from both sides of the zero-energy flow surface are toward it, this surface is an attracting surface.

Figure 3 shows a case where the orbit intersects the energy flow surface at a point $\boldsymbol{y}$. Since $\Delta \dot{E}>0$ above the surface and $\Delta \dot{E}<0$ under the surface, the flow along the orbit is backwards at this point, so this is an unstable point. 
At the fixed point $\boldsymbol{y}=\mathbf{0}$, from Eqs. (15) and (16), we have

$\Delta \dot{E}=\dot{E}(\boldsymbol{\varepsilon})=\boldsymbol{\varepsilon}^{\mathrm{T}} \boldsymbol{E} \boldsymbol{\varepsilon}=\sum_{I=1}^{n} \lambda_{I} \zeta_{I}^{2}$

which implies that the stability of a fixed point $\boldsymbol{y}=\mathbf{0}$ is determined by the energy flow characteristic factors of the energy flow matrix $\boldsymbol{E}$ as in the statement above.

A fixed point $\boldsymbol{y}=\mathbf{0}$ of a nonlinear dynamical system governed by Eq. (1) is asymptotically stable, stable or unstable if the quadratic form of its energy flow in Eq. (17) is definitely negative $(\dot{E}(\boldsymbol{\varepsilon})<0)$, semi-negative $(\dot{E}(\boldsymbol{\varepsilon}) \leq 0)$ or definitely positive $(\dot{E}(\varepsilon)>0)$ in the neighbourhood around the fixed point, respectively, i.e. if the energy flow characteristic factors of the system are all negative $\left(\lambda_{I}<0\right)$ or semi-negative $\left(\lambda_{I} \leq 0\right)$ or there exists at least one positive factor $\left(\lambda_{J}>0\right)$ in the neighbourhood around the fixed point, respectively.

The Lyapunov function method [2] is often used to investigate the stability of fixed points, which relies on finding a positive-definite Lyapunov function. However, finding a Lyapunov function for a generalised nonlinear dynamical system is very difficult. Here, the defined generalised energy $E$ of a nonlinear dynamical system plays the role of a generalised Lyapunov function to investigate the stability of a fixed point of the nonlinear system in the phase space, overcoming this difficulty.

Furthermore, the eigenvalues of the Jacobian matrix in Eq. (6) are widely used to give stability solutions. As is known, the Jacobian matrix is non-symmetrical, with complex eigenvalues. Instead of the non-symmetrical Jacobian matrix, we adopt the real symmetrical energy flow matrix with real eigenvalues to give the stability solution, which is more effective.

It should be mentioned that the statement above about the stability of an equilibrium point is based on a linearised approximation at the zero point; for real linear systems, this conclusion is correct. However, for nonlinear systems, the zero-energy flow characteristic factor cannot guarantee its stability characteristics, and a higher-order approximation analysis is needed. The central energy flow theory discussed in Ref. [1] provides a way to do this and examples to further reveal the stability performance in this case.

\section{Periodical orbit and its energy flow behaviour}

For a nonlinear dynamical system, a periodical orbit is a closed curve in phase space, along which the phase point $\boldsymbol{y}(t)$ with its vector $\boldsymbol{y}(t)$ moves; starting from a position $\boldsymbol{y}(\hat{t})$ and $\dot{\boldsymbol{y}}(\hat{t})$ at time $t=\hat{t}$, it moves to the same position $\boldsymbol{y}(\hat{t}+T)=$ $\boldsymbol{y}(\hat{t})$ and $\dot{\boldsymbol{y}}(\hat{t}+T)=\dot{\boldsymbol{y}}(\hat{t})$ after a time period $T$, and the motion repeats again, as in the case $\hat{t}=0$ shown in Fig. 4 .

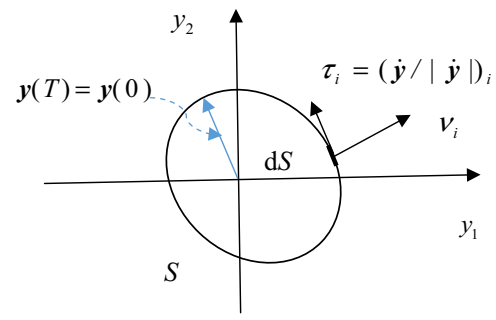

Fig. 4 Periodical orbit and unit normal/tangent vectors of the line element $\mathrm{d} s$ on the orbit

We assume that $\mathrm{d} s$ denotes a differential line element with unit outside normal vector $\boldsymbol{v}$ and unit tangent vector $\boldsymbol{\tau}$ at a point on the closed curve in Fig. 4, so that

$\boldsymbol{\tau} \mathrm{d} s=\dot{\boldsymbol{y}}|\mathrm{d} \boldsymbol{y}| /|\dot{\boldsymbol{y}}|=\dot{\boldsymbol{y}}|\dot{\boldsymbol{y}} \mathrm{d} t| /|\dot{\boldsymbol{y}}|=\dot{\boldsymbol{y}} \mathrm{d} t$,

based on which the following integrals along the curve hold.

\subsection{Time-averaged GPE}

$\langle E\rangle=\frac{1}{T} \int_{0}^{T}\left(\boldsymbol{y}^{\mathrm{T}} \boldsymbol{y} / 2\right) \mathrm{d} t=\hat{E}, \quad$ const.

where $\hat{E}$ is a positive constant relating the averaged distance of the phase points on the orbit to the origin, since the motion repeats along the closed orbit.

\subsection{Time-averaged PEF}

$\langle\dot{E}\rangle=\frac{1}{T} \int_{\hat{t}}^{T+\hat{t}} \dot{E} \mathrm{~d} t=\frac{E(T+\hat{t})-E(\hat{t})}{T}=0$,

since, for the periodical orbit $S, \boldsymbol{y}(T+\hat{t})=\boldsymbol{y}(\hat{t})$.

\subsection{Circulation integral: time-averaged kinetic energy}

$$
\begin{aligned}
\langle K\rangle & =\frac{1}{2 T} \oint_{S} \dot{y} \cdot \boldsymbol{\tau} \mathrm{d} S=\frac{1}{2 T} \oint_{S} \dot{y} \cdot \dot{y} \mathrm{~d} t \\
& =\frac{1}{T} \oint_{S} K \mathrm{~d} t=\hat{K}
\end{aligned}
$$

where $\hat{K}$ is a positive constant, since the phase point periodically moves along the curve and its GKE must be positive at all non-fixed points on the curve. Furthermore, the integral of Eq. (21) implies that $\operatorname{curl} \dot{\boldsymbol{y}}=\operatorname{curl} \boldsymbol{f}$ must not vanish, so we have 
$\langle K\rangle=\frac{1}{T} \oint_{S} K \mathrm{~d} t=\hat{K}, \quad \boldsymbol{U} \neq 0$.

\subsection{Time-averaged kinetic energy flow (KEF)}

$\langle\dot{K}\rangle=\frac{1}{T} \int_{\hat{t}}^{T+\hat{t}} \dot{K} \mathrm{~d} t=\frac{K(T+\hat{t})-K(\hat{t})}{T}=0$,

since for the periodical orbit $S, \dot{\boldsymbol{y}}(T+\hat{t})=\dot{\boldsymbol{y}}(\hat{t})$.

The averaged time rate of change of both the potential and kinetic energies vanishes over a time period, implying that the time-averaged mechanical energy $\langle E+K\rangle$ is conserved over a time period. Therefore, one can say that, for a periodical orbit with time period $T$ in the phase space of a nonlinear dynamical system, its time-averaged mechanical energy is conserved.

\section{Energy flow characteristics of chaotic motions}

Chaos is normally understood as "a state of disorder". Although there is no universally accepted mathematical definition of chaos, a commonly used definition says that, for a dynamical system to be classified as chaotic, it must have the following properties [5]: be sensitive to initial conditions, show topological transitivity, have densely periodical orbits etc. From the energy flow point of view, chaotic motions have these characteristics.

\subsection{Attractor around zero-energy flow surface}

An attractor of a nonlinear dynamical system should be a zero-energy flow surface, towards which the phase points are attracted according to the following energy flow conditions:

$$
\begin{cases}\dot{E}_{d}>0, & d<d_{\dot{E}=0}, \\ \dot{E}_{d}=0, & d=d_{\dot{E}=0}, \\ \dot{E}_{d}<0, & d>d_{\dot{E}=0},\end{cases}
$$

where $d_{\dot{E}=0}$ and $d$ respectively denote the distances of a point $\boldsymbol{y}$ on the zero-energy flow surface and a neighbouring point around this point $\boldsymbol{y}$ to the origin of phase space.

\subsection{Negative time rate of change of phase volume strain}

Flows are restricted to a finite volume, so that the averaged time rate of change of the phase volume strain of the phase space must not be positive; i.e. from Eq. (12), we have

$\dot{v}_{V}=\frac{1}{V} \int_{V} \dot{v} \mathrm{~d} V=\frac{1}{V} \int_{V} \sum_{I=1}^{n} \lambda_{I} \mathrm{~d} V \leq 0$.

\subsection{Periodical motion with infinite time period}

Based on the above characteristics, a chaotic motion can be considered as a periodical motion with infinite time period, so that it should have the characteristics of a periodical motion with infinite time period. From Eqs. (19)-(23) valid for periodical orbits, actually using the integral mean value theorem [6], we obtain

$$
\begin{aligned}
\langle E\rangle & =\lim _{T \rightarrow \infty}\left(\frac{1}{T} \int_{\hat{t}}^{T+\hat{t}} E \mathrm{~d} t\right) \\
& =\lim _{T \rightarrow \infty}\left(\frac{T \hat{E}}{T}\right) \rightarrow \hat{E}, \quad \text { const., } \\
\langle\dot{E}\rangle & =\lim _{T \rightarrow \infty}\left(\frac{1}{T} \int_{\hat{t}}^{T+\hat{t}} \dot{E} \mathrm{~d} t\right) \rightarrow 0, \\
\langle K\rangle & =\lim _{T \rightarrow \infty}\left(\frac{1}{T} \int_{\hat{t}}^{T+\hat{t}} K \mathrm{~d} t\right) \\
& =\lim _{T \rightarrow \infty}\left(\frac{T \hat{K}}{T}\right) \rightarrow \hat{K}, \quad \text { const., } \\
\langle\dot{K}\rangle & =\lim _{T \rightarrow \infty}\left(\frac{1}{T} \int_{\hat{t}}^{T+\hat{t}} \dot{K} \mathrm{~d} t\right) \rightarrow 0 .
\end{aligned}
$$

It can thus be concluded that a chaotic motion of a nonlinear dynamical system can be considered as a periodical motion with infinite time period $T \rightarrow \infty$ whose time-averaged generalised energies are conserved with increasing averaging time.

\section{Numerical investigation}

Using Matlab software [3], the chaotic motion of nonlinear dynamical systems reported worldwide, such as the forced van der Pol system [7], Duffing system [8], Lorenz system [9, 10], Rössler system [11] and smooth and discontinuous (SD) oscillator [12-14], is investigated. The numerical results are shown in Figs. 6-10, using the following notations:

(I) a Phase diagram and $\mathbf{b}$ time rate of change of phase volume strain;

(II) a Time histories of GPE and b PEF, the curves of c time-averaged GPE versus the averaging time $T$ and d $\mathrm{d} E / \mathrm{d} t$ versus the averaging time $T$;

(III) a Time histories of GKE and $\mathbf{b} \mathrm{KEF}$, the curves of c time-averaged GKE versus the averaging time $T$ and d $\mathrm{d} K / \mathrm{d} t$ versus the averaging time $T$.

All the numerical results demonstrate that the energy characteristics of the chaotic motions of these nonlinear dynam- 
ical systems are correct. Here, as an example, a detailed discussion of the Lorenz system is presented, neglecting the related discussions for the other systems except listing the governing equations with the used parameters.

Lorenz system: $\alpha=10, \gamma=8 / 3, \beta=28$ [10],

$\dot{\boldsymbol{y}}=\boldsymbol{f}, \quad\left\{\begin{array}{l}\dot{x}=\alpha(y-x), \\ \dot{y}=\beta x-y-x z, \\ \dot{z}=-\gamma z+x y,\end{array}\right.$

$\alpha, \beta, \gamma>0, \quad \boldsymbol{y}=\left[\begin{array}{l}x \\ y \\ z\end{array}\right]$

for which the Jacobian matrix, energy flow and spin matrix are respectively

$\boldsymbol{J}=\left[\begin{array}{ccc}-\alpha & \alpha & 0 \\ \beta-z & -1 & -x \\ y & x & -\gamma\end{array}\right]$,

$\boldsymbol{E}=\left[\begin{array}{ccc}-\alpha & (\alpha+\beta-z) / 2 & y / 2 \\ (\alpha+\beta-z) / 2 & -1 & 0 \\ y / 2 & 0 & -\gamma\end{array}\right]$,

$\boldsymbol{U}=\left[\begin{array}{ccc}0 & (\alpha-\beta+z) / 2-y / 2 \\ -(\alpha-\beta+z) / 2 & 0 & -x \\ y / 2 & x & 0\end{array}\right]$.

\subsection{Energy flow equation and characteristic factors}

The energy flow equation of the Lorenz equation is

$$
\begin{aligned}
& \dot{E}=\left[\begin{array}{lll}
x & y & z
\end{array}\right] \boldsymbol{f}=\left[\begin{array}{lll}
x & y & z
\end{array}\right] \boldsymbol{E}_{0}\left[\begin{array}{lll}
x & y & z
\end{array}\right]^{\mathrm{T}} \text {, } \\
& \boldsymbol{E}_{0}=\boldsymbol{E}(x=y=z=0) \\
& =\left[\begin{array}{ccc}
-\alpha & (\alpha+\beta) / 2 & 0 \\
(\alpha+\beta) / 2 & -1 & 0 \\
0 & 0 & -\gamma
\end{array}\right] \text {. }
\end{aligned}
$$

For different bifurcation parameters, the energy flow matrix has the following three energy flow characteristic factors:

$$
\begin{aligned}
& \lambda_{1}=\frac{-(\alpha+1)+B}{2}\left\{\begin{array}{l}
<0, \beta<2 \sqrt{\alpha}-\alpha, \\
=0, \beta=2 \sqrt{\alpha}-\alpha, \\
>0, \beta>2 \sqrt{\alpha}-\alpha,
\end{array}\right. \\
& \lambda_{2}=\frac{-(\alpha+1)-B}{2}<0, \quad \lambda_{3}=-\gamma<0, \\
& B=\sqrt{(\alpha+1)^{2}+(\alpha+\beta)^{2}-4 \alpha .}
\end{aligned}
$$

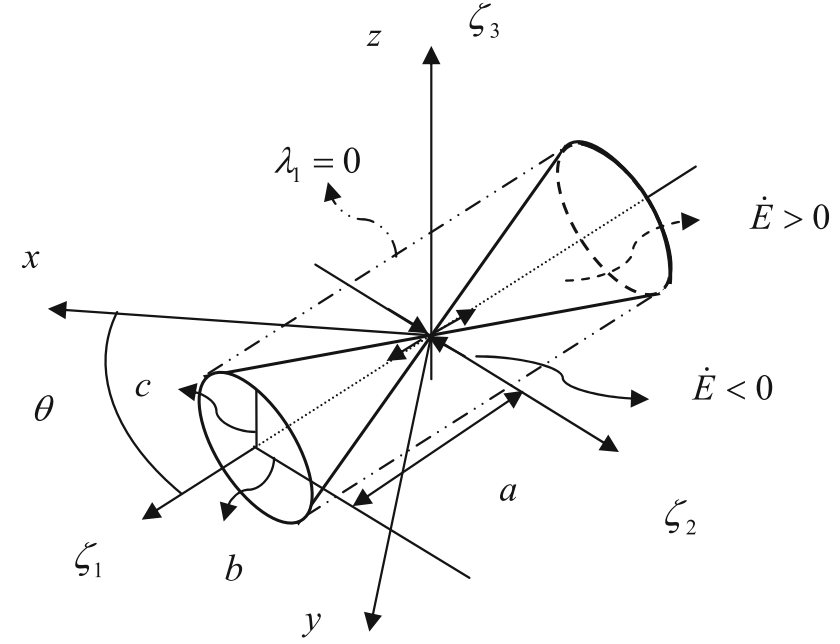

Fig. 5 Zero-energy flow surface governed by $\dot{E}=0$ from Eq. (36) in which $\lambda_{1}>0, \lambda_{2}>0$ and $\lambda_{3}>0$. Here $a=1 / \sqrt{\lambda_{1}}, b=1 / \sqrt{-\lambda_{2}}$ and $c=1 / \sqrt{-\lambda_{3}}$. The long-dash double-dot lines define an elliptical cylinder for the case $\beta=1, \alpha=1$ and $\lambda_{1}=0$

\subsection{Zero-energy flow surface and its bifurcation}

The energy flow Eq. (34) is represented in the energy flow space $o-\zeta_{1} \zeta_{2} \zeta_{3}$ span by the energy flow characteristic vectors in the form

$\dot{E}=\lambda_{1} \zeta_{1}^{2}+\lambda_{2} \zeta_{2}^{2}+\lambda_{3} \zeta_{3}^{2}$

from which the zero-energy flow surface $\dot{E}=0$ and its bifurcation depending on the different parameter values can be obtained. The work of Lanford [10] revealed the strange attractor of a Lorenz system with parameters $\alpha=10, \gamma=$ $8 / 3$ and $\beta=28$, which, by Eq. (35), has one positive $\left(\lambda_{1}>0\right)$ and two negative $\left(\lambda_{2}<0, \lambda_{3}<0\right)$ energy flow factors, so that its zero-energy flow surface is as shown in Fig. 5. Due to the two negative energy flow factors, in the interior of the surface, the energy flow $\dot{E}>0$, while outside the surface, it is $\dot{E}<0$. Therefore, the flows at points outside the surface move towards the origin to reduce the potential while the flows in the interior of surface move backwards the origin to increase the potential. As result of this, this surface would be an attracting surface.

\subsection{Equilibrium points and stabilities}

\subsubsection{Origin $(0,0,0)$}

The origin $(0,0,0)$ is a fixed point, being globally stable if $\beta \leq 2 \sqrt{\alpha}-\alpha$ and unstable if $\beta>2 \sqrt{\alpha}-\alpha$, since there are three negative energy flow factors for the stable case but one positive factor $\lambda_{1}>0$ for the unstable case. 
(a) Phase digram

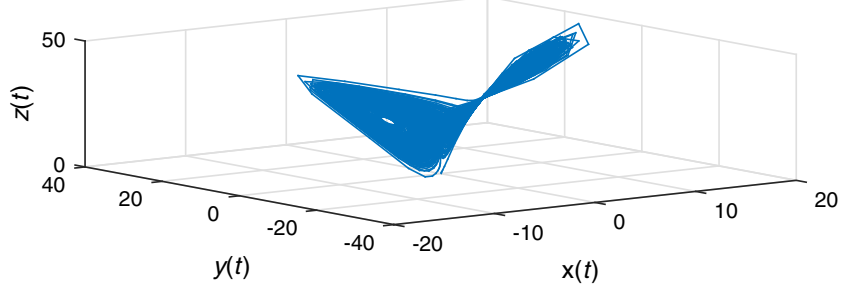

(b) Phase space volume strain

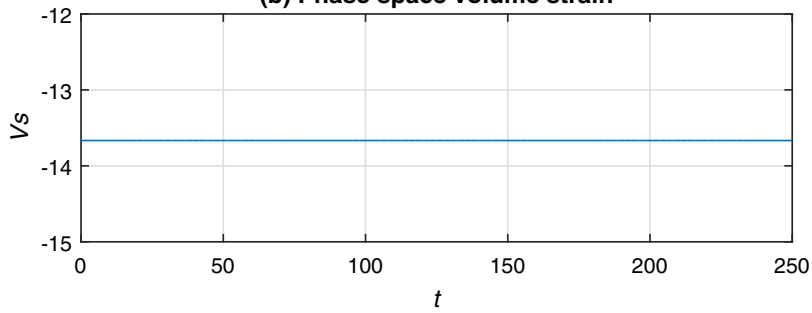

(a) Time history of potential energy
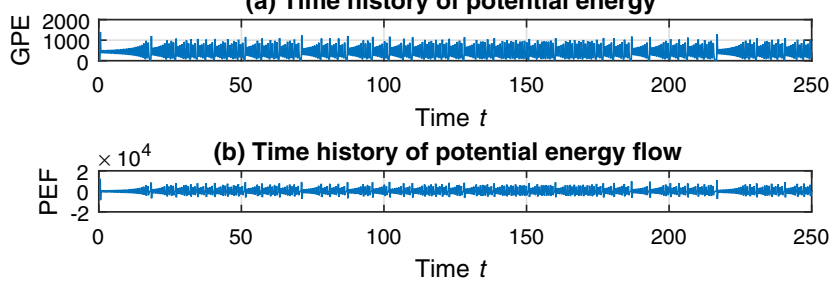

(c) Time averaged potential energy
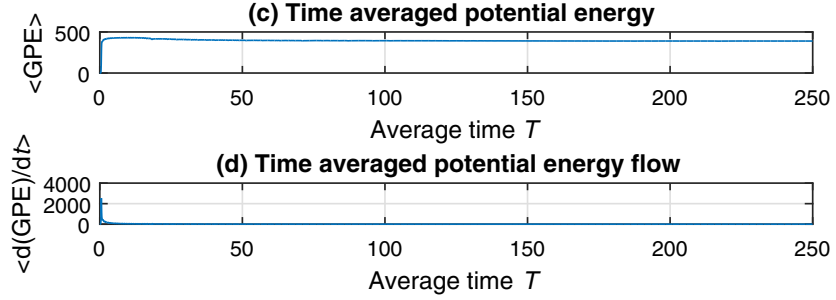
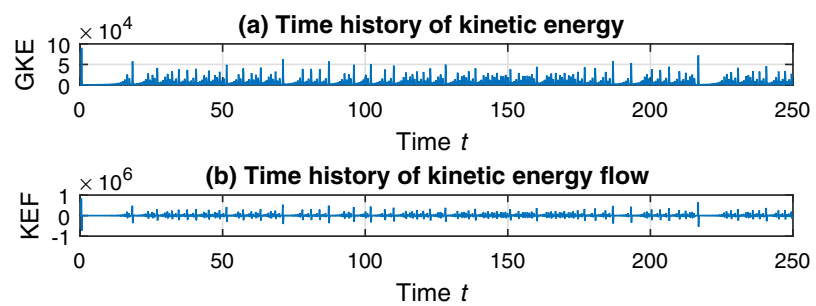

(c) Time averaged kinetic energy
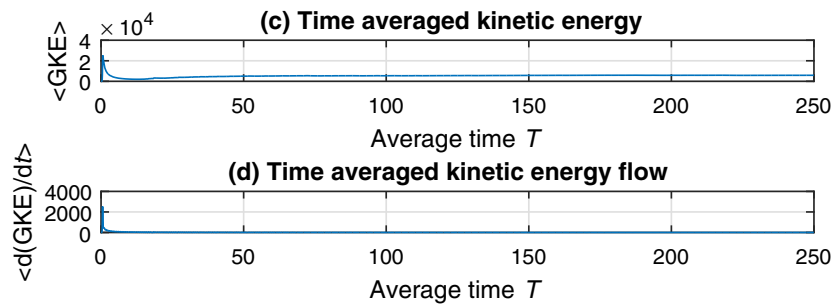

Fig. 6 Chaotic motion of Lorenz system $(\alpha=10, \gamma=8 / 3$ and $\beta=28)$, GPE means generalised potential energy $E$, GKE means generalised kinetic energy $K$

\subsubsection{Nontrivial points}

In the condition $x=y$ and $\beta>1$, there are two fixed points:

$x= \pm \sqrt{\gamma(\beta-1)}=y, \quad z=\beta-1$,

at which $\dot{E}=0$. Since in the case $\beta>1, \beta>2 \sqrt{\alpha}-\alpha$ is valid, these two fixed points are unstable. When $\beta \rightarrow 1$, these two points tend to the origin.

\subsection{Energy flow characteristics of chaotic motion}

\subsubsection{Time rate of change of phase volume strain}

The time rate of change of the phase volume strain in this Lorenz system can be obtained by using Eqs. (12) and (31), i.e.
$\dot{v}=\operatorname{tr}(\boldsymbol{E})=\sum_{I=1}^{3} \lambda_{I}=-1-\alpha-\gamma<0$,

which holds at all points on the orbit; therefore, the phase volume of this Lorenz system is in contraction at every point in phase space. Figure 6(I) shows the phase diagram and the time rate of change of the phase volume strain obtained by numerical simulation for this system with the initial condition $(0.1,0.1,0)$, which shows a type of attractor similar to that in Fig. 5 and a constant negative time rate of change of the phase volume strain.

\subsubsection{Time-averaged potential energy and its flow}

From Eqs. (27) and (36), the time-averaged potential energy flow of this Lorenz system is given by

$\langle\dot{E}\rangle=\lim _{T \rightarrow \infty}\left(\frac{1}{T} \int_{\hat{t}}^{T+\hat{t}} \dot{E} \mathrm{~d} t\right)$ 


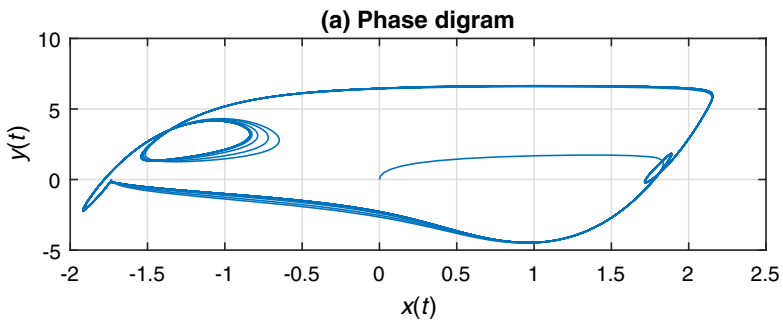

(b) Phase space volume strain

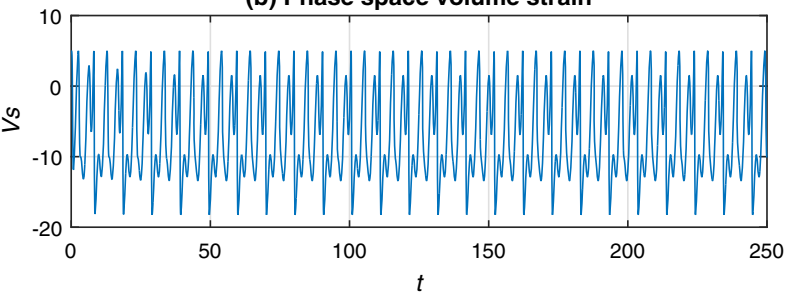

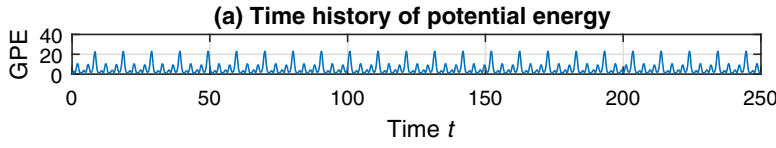

(b) Time history of potential energy flow
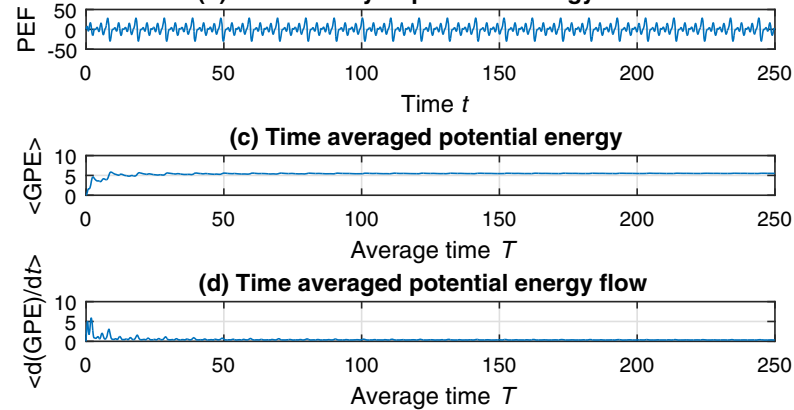

(a) Time history of kinetic energy

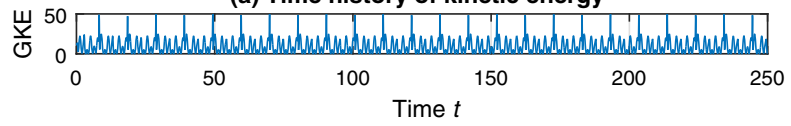

(b) Time history of kinetic energy flow

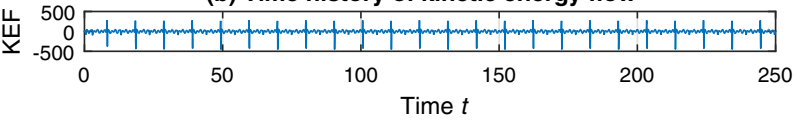

(c) Time averaged kinetic energy
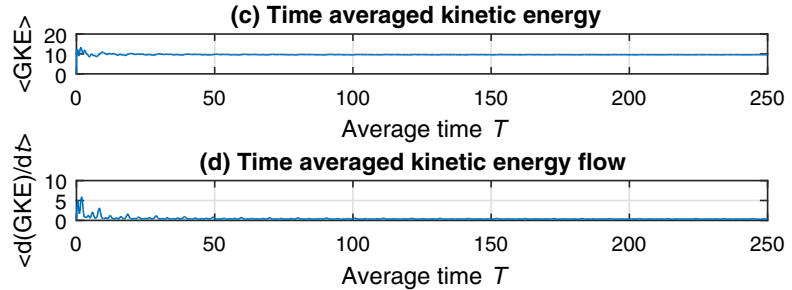

Fig. 7 Chaotic motion of forced van der Pol system ( $\alpha=5, F=5, \omega=2.446, \varphi=0)$, GPE means generalised potential energy $E$, GKE means generalised kinetic energy $K$

$$
=\lim _{T \rightarrow \infty}\left(\frac{1}{T} \int_{\hat{t}}^{T+\hat{t}} \sum_{I=1}^{3} \lambda_{I} \zeta_{I}^{2} \mathrm{~d} t\right) \rightarrow 0 .
$$

Due to the one positive factor $\lambda_{1}$ and two negative factors $\lambda_{2}$ and $\lambda_{3}$ with the orbit restricted to a finite phase volume as shown by Fig. 6(I), the value of Eq. (39) tends to zero when $T \rightarrow \infty$. As shown in Fig. 6(II), the time history of GPE oscillates around a positive mean value, so that the time-averaged GPE tends to a constant mean value when the averaging time $T \rightarrow \infty$, while the PEF oscillates around a zero mean value and its time-averaged $\mathrm{d} E / \mathrm{d} t$ tends to zero when the averaging time $T \rightarrow \infty$.

\subsubsection{Time-averaged kinetic energy and its flow}

Using the time rate of change of the kinetic energy defined by Eq. (6) in Eq. (30) gives

$\dot{K}=\dot{\boldsymbol{y}}^{\mathrm{T}} \ddot{\boldsymbol{y}}=\dot{\boldsymbol{y}}^{\mathrm{T}} \boldsymbol{J} \dot{\boldsymbol{y}}=\dot{\boldsymbol{y}}^{\mathrm{T}} \boldsymbol{E} \dot{\boldsymbol{y}}=\dot{\boldsymbol{y}}^{\mathrm{T}} \boldsymbol{E}_{0} \dot{\boldsymbol{y}}+A$,

$$
\begin{aligned}
A & =\dot{\boldsymbol{y}}^{\mathrm{T}}\left[\begin{array}{ccc}
0 & -z / 2 & y / 2 \\
-z / 2 & 0 & 0 \\
y / 2 & 0 & 0
\end{array}\right] \dot{\boldsymbol{y}} \\
& =\dot{x}[\dot{y} \dot{z}] \overline{\boldsymbol{U}}\left[\begin{array}{l}
y \\
z
\end{array}\right], \quad \overline{\boldsymbol{U}}=\left[\begin{array}{cc}
0 & -1 \\
1 & 0
\end{array}\right] .
\end{aligned}
$$

The eigenvectors of the spin matrix $\bar{U}$ are

$\boldsymbol{\Phi}=\frac{1}{\sqrt{2}}\left[\begin{array}{cc}1 & 1 \\ -\mathrm{i} & \mathrm{i}\end{array}\right]$

based on which, without loss of generality, the real vector $\left[\begin{array}{ll}y & z\end{array}\right]^{\mathrm{T}}$ and its time derivative can be decomposed into the complex forms

$\left[\begin{array}{l}y \\ z\end{array}\right]=\boldsymbol{\Phi}\left[\begin{array}{l}\tilde{\xi}_{2} \\ \tilde{\xi}_{3}\end{array}\right] \mathrm{e}^{\mathrm{i} \theta(t)}, \quad\left[\begin{array}{l}\dot{y} \\ \dot{z}\end{array}\right]=\mathrm{i} \dot{\theta} \boldsymbol{\Phi}\left[\begin{array}{l}\tilde{\xi}_{2} \\ \tilde{\xi}_{3}\end{array}\right] \mathrm{e}^{\mathrm{i} \theta(t)}$, 


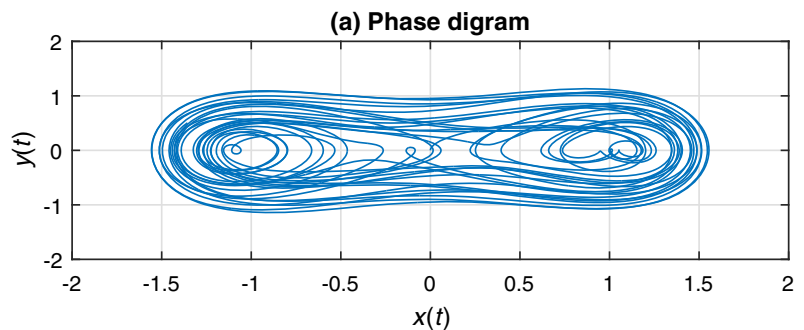

(b) Phase space volume strain

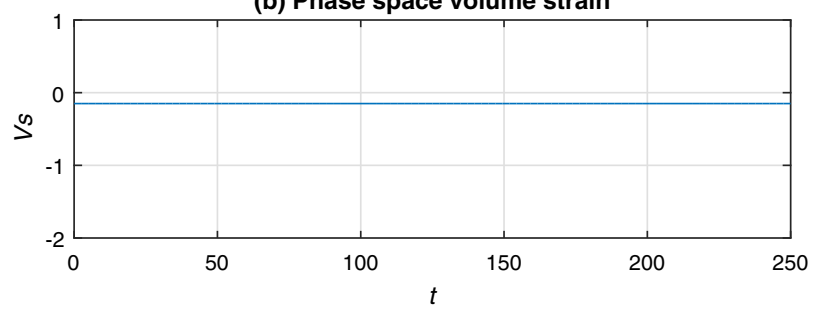

(a) Time history of potential energy

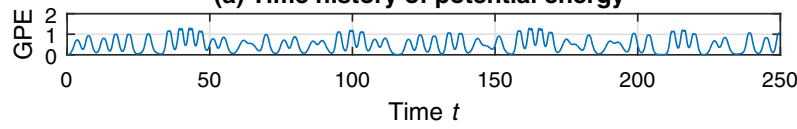

(b) Time history of potential energy flow

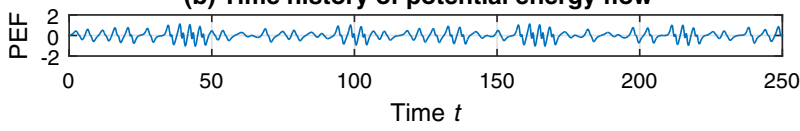

(c) Time averaged potential energy
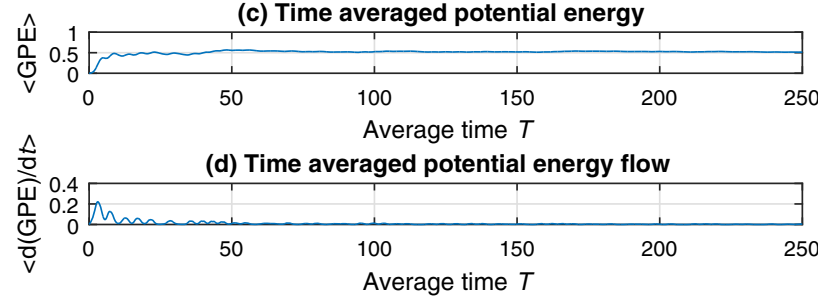

(a) Time history of kinetic energy

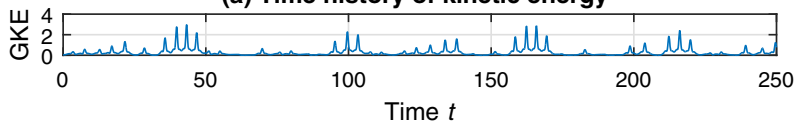

(b) Time history of kinetic energy flow

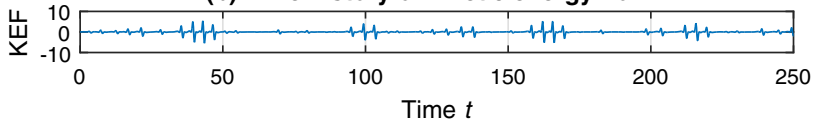

(c) Time averaged kinetic energy
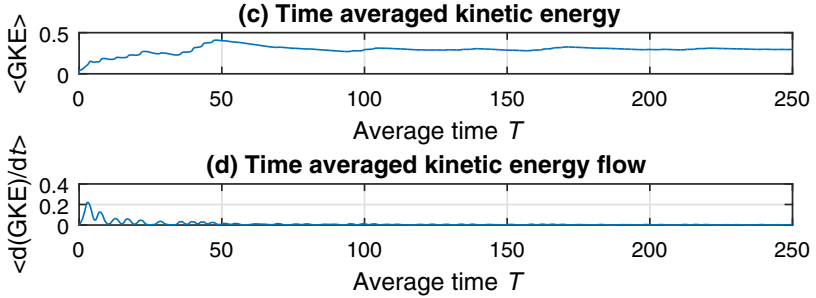

Fig. 8 Chaotic motion of forced Duffing system $(F=0.3, \omega=1.0, \delta=0.15, \varphi=0)$, GPE means generalised potential energy $E$, GKE means generalised kinetic energy $K$

since the spin matrix $\overline{\boldsymbol{U}}$ corresponds to a rotation $\theta(t)[3]$. As a result of this, we obtain that

$$
\begin{aligned}
& {\left[\begin{array}{ll}
\dot{y} \dot{z}
\end{array}\right] \overline{\boldsymbol{U}}\left[\begin{array}{l}
y \\
z
\end{array}\right]=-\mathrm{i} \dot{\theta} \mathrm{e}^{-\mathrm{i} \theta(t)}\left[\begin{array}{ll}
\tilde{\xi}_{2}^{*} & \tilde{\xi}_{3}^{*}
\end{array}\right] \boldsymbol{\Phi}^{* \mathrm{~T}} \overline{\boldsymbol{U}} \boldsymbol{\Phi}\left[\begin{array}{l}
\tilde{\xi}_{2} \\
\tilde{\xi}_{3}
\end{array}\right] \mathrm{e}^{\mathrm{i} \theta(t)}} \\
& =\dot{\theta}\left(\tilde{\xi}_{2}^{*} \tilde{\xi}_{2}-\tilde{\xi}_{3}^{*} \tilde{\xi}_{3}\right)=\dot{\theta}\left(\left|\tilde{\xi}_{2}\right|^{2}-\left|\tilde{\xi}_{3}\right|^{2}\right) \text {, real number. }
\end{aligned}
$$

Therefore, Eq. (40) becomes

$$
\begin{aligned}
\dot{K} & =\dot{\mathbf{y}}^{\mathrm{T}} \boldsymbol{E}_{0} \dot{\boldsymbol{y}}+\dot{x} \dot{\theta}\left(\left|\tilde{\xi}_{2}\right|^{2}-\left|\tilde{\xi}_{3}\right|^{2}\right) \\
& =\sum_{I=1}^{3} \lambda_{I} \dot{\zeta}_{I}^{2}+\dot{x} \dot{\theta}\left(\left|\tilde{\xi}_{2}\right|^{2}-\left|\tilde{\xi}_{3}\right|^{2}\right),
\end{aligned}
$$

whose, in a similar way to Eq. (39), time-averaged value

$\langle\dot{K}\rangle=\lim _{T \rightarrow \infty}\left(\frac{1}{T} \int_{\hat{t}}^{T+\hat{t}} \dot{K} \mathrm{~d} t\right) \rightarrow 0$.

The curves in Fig. 6(III) show that the time histories of the GKE and KEF oscillate respectively around their mean values, while the time-averaged $\mathrm{GKE}$ and $\mathrm{d} K / \mathrm{d} t$ respectively tend to a constant and zero as the averaging time $T \rightarrow \infty$.

\subsection{Forced van der Pol system}

$\alpha=5, F=5, \omega=2.446, \varphi=0[7]$,

$\left[\begin{array}{l}\dot{y}_{1} \\ \dot{y}_{2}\end{array}\right]=\left[\begin{array}{c}y_{2}-\alpha\left(y_{1}^{3} / 3-y_{1}\right) \\ -y_{1}\end{array}\right]+\left[\begin{array}{c}0 \\ F \cos (\omega t+\varphi)\end{array}\right]$.

Figure 7 shows the numerical simulation results. 
(a) Phase digram

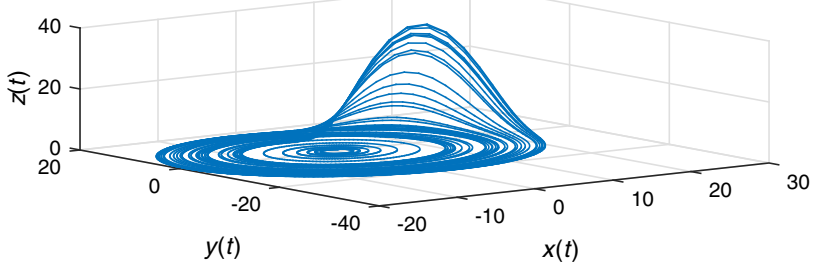

(b) Phase space volume strain

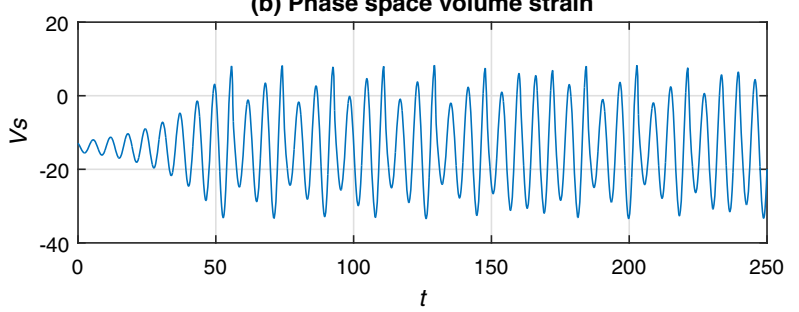

(a) Time history of potential energy

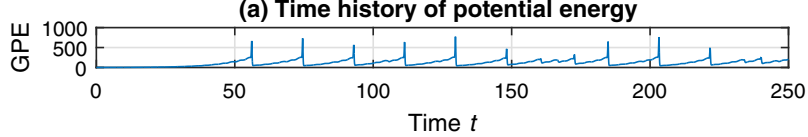

(b) Time history of potential energy flow

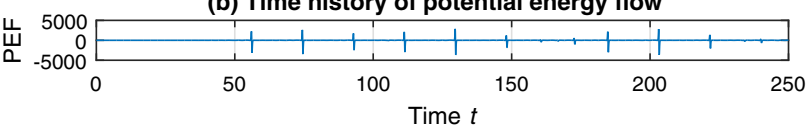

(c) Time averaged potential energy
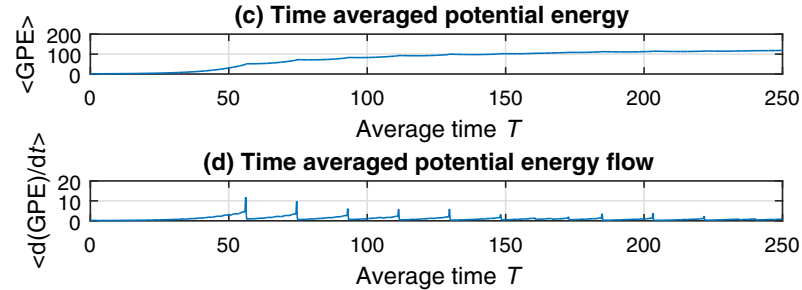

(a) Time history of kinetic energy
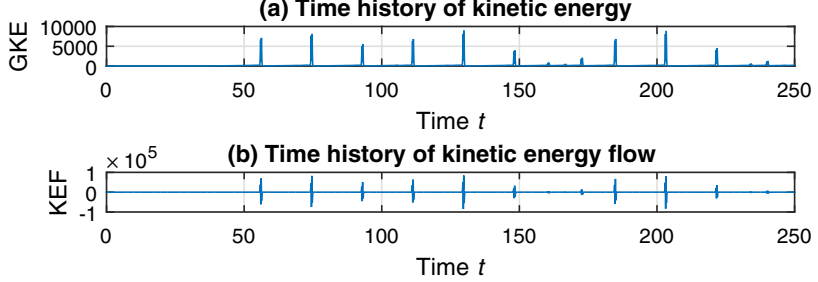

(c) Time averaged kinetic energy
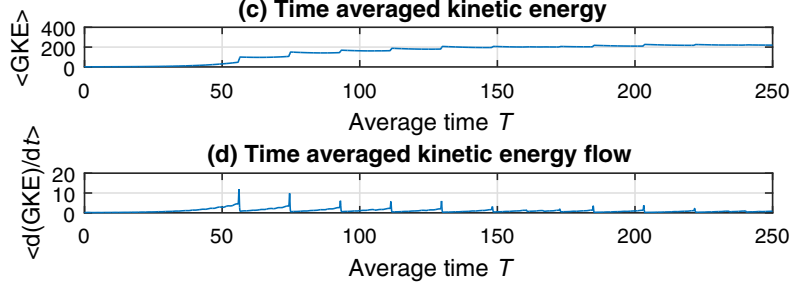

Fig. 9 Chaotic motion of Rössler system $(\alpha=0.1=\beta, \gamma=14)$ with initial condition (1.0, 1.0, 0), GPE means generalised potential energy $E$, GKE means generalised kinetic energy $K$

\subsection{Forced Duffing system}

$F=0.3, \omega=1.0, \delta=0.15, \varphi=0[8]$,

$\left[\begin{array}{l}\dot{y}_{1} \\ \dot{y}_{2}\end{array}\right]=\left[\begin{array}{c}y_{2} \\ y_{1}-y_{1}^{3}-\delta y_{2}\end{array}\right]+\left[\begin{array}{c}0 \\ F \cos (\omega t+\varphi)\end{array}\right]$.

The simulation results are shown in Fig. 8.

\subsection{Rössler system}

$\alpha=0.1=\beta, \gamma=14[11]$,

$\left[\begin{array}{l}\dot{y}_{1} \\ \dot{y}_{2} \\ \dot{y}_{3}\end{array}\right]=\left[\begin{array}{c}-\left(y_{2}+y_{3}\right) \\ y_{1}+\alpha y_{2} \\ \beta+y_{3}\left(y_{1}-\gamma\right)\end{array}\right],\left[\begin{array}{l}y_{1} \\ y_{2} \\ y_{3}\end{array}\right]_{0}=\left[\begin{array}{c}1.0 \\ 1.0 \\ 0\end{array}\right]$.

The simulation results are shown in Fig. 9.

\subsection{Forced SD oscillator}

$\alpha=0.01, F=0.8, \delta=1, \omega=1.0605, \gamma=0.01415$, $g=0.5[12,13]$,

$\left[\begin{array}{l}\dot{y}_{1} \\ \dot{y}_{2}\end{array}\right]=\left[\begin{array}{c}y_{2} \\ -2 \gamma y_{2}-y_{1}\left(1-\frac{1}{\sqrt{y_{1}^{2}+\alpha^{2}}}-\delta g\right)\end{array}\right]$

$+\left[\begin{array}{c}0 \\ F \cos (\omega t+\varphi)\end{array}\right]$.

The simulation results are shown in Fig. 10.

\section{Conclusions and discussion}

Two real scalars, viz. the generalised potential and kinetic energies, are defined in phase space to investigate the dynamical behaviour of nonlinear dynamical systems. The GPE 


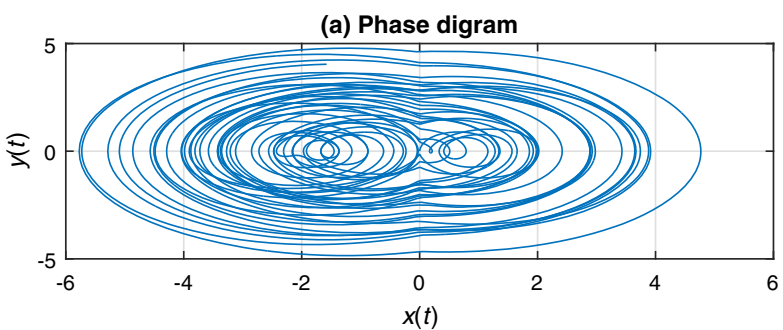

(b) Phase space volume strain

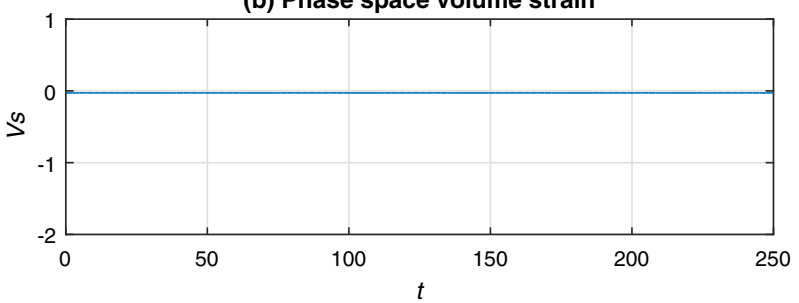

(a) Time history of potential energy

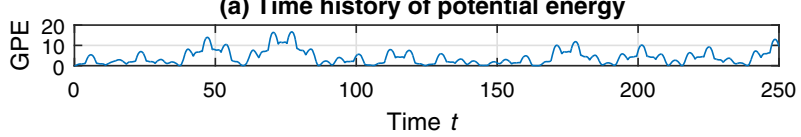

(b) Time history of potential energy flow

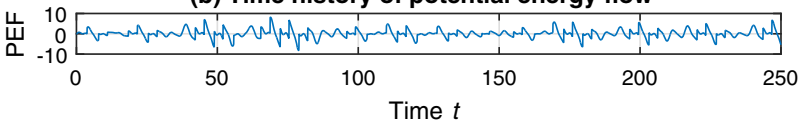

(c) Time averaged potential energy
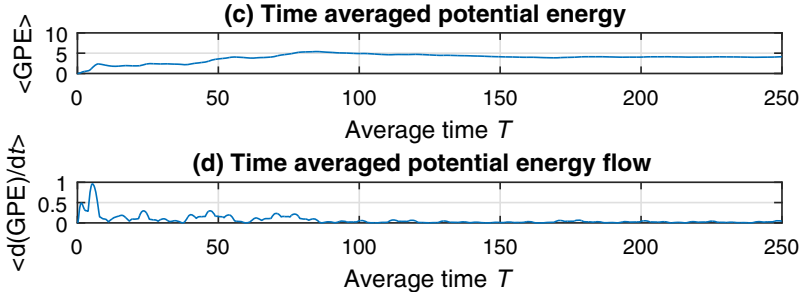

(a) Time history of kinetic energy

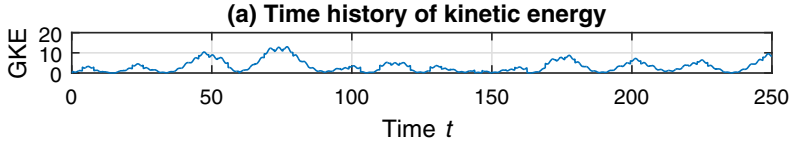

(b) Time history of kinetic energy flow

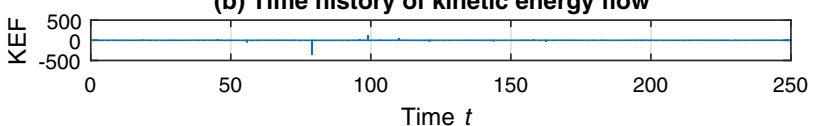

(c) Time averaged kinetic energy
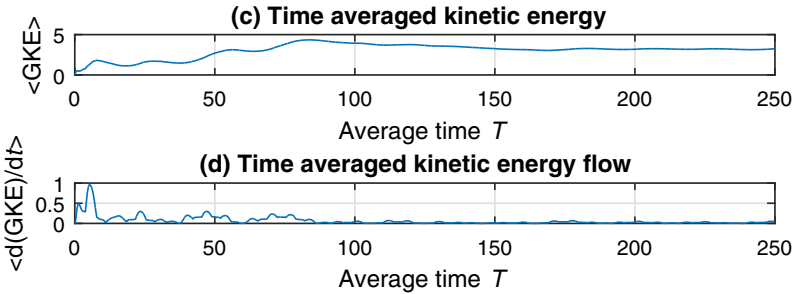

Fig. 10 Chaotic motion of forced SD oscillator $(\alpha=0.01, F=0.8, \omega=1.0605, \gamma=0.01415, \delta=1, g=0.5)$, GPE means generalised potential energy $E$, GKE means generalised kinetic energy $K$

plays the role of a Lyapunov function suitable for all nonlinear dynamical systems to examine the stability about fixed points, while the real symmetrical energy flow matrix can replace the non-symmetrical Jacobian matrix to investigate the behaviour of the linearised approximation at a point of a nonlinear system. The chaotic motion of a nonlinear dynamical system with a negative time rate of change of the phase volume strain is restricted to finite regions of phase space, so they can be considered as periodical motions with infinite time period. As the averaging time tends to infinite, the time-averaged potential and kinetic energies of chaotic motions tend to constants, while the time-averaged potential and kinetic energy flows vanish, providing an energy conservation law for chaotic motions in nature. Numerical simulations giving curves for the chaotic motion of five worldwide recognised nonlinear dynamical systems demonstrate that the above conclusions are correct.
It has been noted that (1) quasi-periodical motions are sometimes considered to be motions with infinite time period. For example, a motion consisting of two harmonic motions with an irrational ratio of their frequencies has no common finite time period, so its time period is considered to be infinite. Concerning the relationship between chaotic motions and quasi-periodical motions, Wang and Hao [15] revealed the transition from quasi-periodical to chaotic motions; (2) the Casimir power of the Qi chaotic system [16] shows the same form of the GPE developed in Ref. [3] and used in this paper, which has been employed to uncover the mechanism of various dynamical systems; see Refs. [16]-[19]. These evidences further confirm applications of the developed generalised energy flow theory. In principle, any nonlinear dynamical system defined by Eq. (1) can be investigated using the developed energy flow theory to reveal their unknown phenomena. 
Acknowledgements The author expresses his thanks to the referee for providing Refs. [16]-[19] on Casimir power used to investigate nonlinear dynamical systems.

Open Access This article is distributed under the terms of the Creative Commons Attribution 4.0 International License (http://creativecomm ons.org/licenses/by/4.0/), which permits unrestricted use, distribution, and reproduction in any medium, provided you give appropriate credit to the original author(s) and the source, provide a link to the Creative Commons license, and indicate if changes were made.

\section{References}

1. Guckenheimer, J., Holmes, P.: Nonlinear Oscillations, Dynamical Systems, and Bifurcations of Vector Fields. Springer, New York (1983)

2. Hirsch, M.W., Smale, S.: Differential Equations, Dynamical Systems and Linear Algebra. Academic, New York (1974)

3. Xing, J.T.: Energy Flow Theory of Nonlinear Dynamical Systems with Applications. Springer, New York (2015)

4. Fung, Y.C.: A First Course in Continuum Mechanics. PrenticeHall, Englewood Cliffs (1977)

5. Hasselblatt, B., Katok, A.: A First Course in Dynamics: With a Panorama of Recent Developments. Cambridge University Press, Oxford (2003)

6. Rudin, W.: Principles of Mathematical Analysis, 3rd edn. McGraw Hill, New York (1976)

7. Parlitz, U., Lauterborn, W.: Period-doubling cascades and Devil's staircases of van der Pol oscillator. Phys. Rev. A 36, 1428-1434 (1987)
8. Moon, F.C., Holmes, P.J.: A magnetoelastic strange attractor. J. Sound Vib. 65(2), 285-296 (1979)

9. Williams, R.F.: The structure of Lorenz attractors. Publications Mathematiques de l'IHES 50, 73-99 (1979)

10. Lanford, O.E.: Computer pictures of Lorenz attractor. Appendix to lecture VII. In: Turbulence Seminar, pp. 113-116. Springer, Berlin (1977)

11. Rössler, O.E.: An equation for continuous chaos. Phys. Lett. A 57(5), 397-398 (1976)

12. Cao, Q., Wiercigroch, M., Pavlovskaia, E.E., et al.: SD oscillator, SD attractor and its applications. J. Vib. Eng. 20(5), 454-458 (2007)

13. Cao, Q., Wiercigroch, M., Pavlovskaia, E.E., et al.: Piecewise linear approach to an archetypal oscillator for smooth and discontinuous dynamics. Philos. Trans. R. Soc. Lond. A 366, 635-652 (2008)

14. Cao, Q., Leger, A.: A Smooth and Discontinuous Oscillator: Theory, Methodology and Applications. Springer, Berlin (2017)

15. Wang, G., Hao, B.: Transition from quasi-periodical motion to chaotic motion. Nat. Mag. 7(6), 474 (1984). (in Chinese)

16. Qi, G., Zhang, J.: Energy cycle and bound of Qi chaotic system. Chaos Solition. Fract. 99, 7-15 (2017)

17. Qi, G., Liang, X.: Mechanism and energy cycling of Qi four-wing chaotic system. Int. J. Bifurc. Chaos 27, 1750180 (2017)

18. Qi, G., Hu, J.: Force analysis and energy operation of chaotic system of permanent-magnet synchronous motor. Int. J. Bifurc. Chaos 27, 1750216 (2017)

19. Qi, G.: Modelings and mechanism analysis underlying both the 4D Euler equations and Hamiltonian conservative chaotic systems. Nonlin. Dyn. 95, 2063-2077 (2019) 